\title{
Biocompósitos de Acetato de Celulose e Fibras Curtas de Curauá Tratadas com $\mathrm{CO}_{2}$ Supercrítico
}

\author{
Miguel C. Gutiérrez, Paulo de T. V. e Rosa, Marco-Aurelio De Paoli, Maria I. Felisberti \\ Instituto de Química, UNICAMP
}

\begin{abstract}
Resumo: Neste trabalho foram desenvolvidos biocompósitos baseados em acetato de celulose e fibras curtas de Curauá tratadas com dióxido de carbono supercrítico. O tratamento das fibras resultou na extração parcial de lignina, sendo este um método interessante pois não resulta em rejeitos químicos. Duas séries de biocompósitos, uma delas plastificada com ftalato de dioctila (DOP) e outra com citrato de trietila (TEC), foram preparadas por extrusão. Para ambas ocorreu a fibrilação e distribuição uniforme das fibrilas. Como consequiência, os biocompósitos apresentaram maior capacidade calorífica, menor condutividade térmica e maior coeficiente de expansão térmica em comparação ao acetato de celulose plastificado. $\mathrm{O}$ tratamento das fibras $\operatorname{com} \mathrm{CO}_{2}$ supercrítico intensificou as variações destas propriedades. Dentre os plastificantes, o DOP mostrou-se ligeiramente mais eficiente, resultando em materiais com menores valores de Tg e de módulo de Young. A adição das fibras teve um impacto relativamente baixo sobre o módulo (10\%), porém houve uma perda significativa da resistência ao impacto. O conjunto de resultados permite concluir que estes biocompósitos apresentam potencial de aplicação como isolantes térmicos, sendo que os plastificados com TEC apresentam como vantagem o fato de todos seus componentes serem biodegradáveis.
\end{abstract}

Palavras-chave: Biocompósitos, fibras lignocelulósicas, polímeros biodegradáveis, fluídos supercríticos, condutividade térmica.

\section{Biocomposites Based on Cellulose Acetate and Short Curaua Fibers Treated with Supercritical $\mathrm{CO}_{2}$}

Abstract: In this work, the effect of pre-processing of short Curaua fibers with supercritical carbon dioxide on the properties of biocomposites with cellulose acetate was studied. The treatment with supercritical $\mathrm{CO}_{2}$ may result in the partial lignin extraction from the fibers. Two series of biocomposites, one plasticized with dioctyl phtalate (DOP) and another with triethyl citrate (TEC), were prepared by extrusion. Fibrilation and uniform distribution of fibers in the cellulose acetate matrix were observed for both biocomposites. As a consequence, the composites showed a higher specific heat, lower thermal conductivity and higher coefficient of thermal expansion than plasticized cellulose acetate. The treatment of the fibers increased such differences. Among the plasticizers, DOP was more efficient, decreasing Tg and Young's modulus of plasticized cellulose acetate. The fiber addition had a relatively low impact on the modulus (10\%), however caused a decrease in the impact resistance. Taken together, the results show that these biocomposites are promising as thermal insulators, with the additional advantage of biodegradability of all components in the case of biocomposites plasticized with TEC.

Keywords: Biocomposites, lignocellulosic fibers, supercritical fluids, biodegradable polymers, thermal conductivity.

\section{Introdução}

Tradicionalmente os compósitos baseados em termoplásticos disponíveis no mercado são constituídos por poliolefinas (principalmente polietileno e polipropileno) e fibras de vidro. As propriedades mecânicas e térmicas destes compósitos permitem aplicações nas indústrias automotiva e aeroespacial ${ }^{[1]}$. Os compósitos disponíveis no mercado apresentam um tempo longo de degradação e, para várias aplicações, estes produtos poliméricos podem ter uma vida útil relativamente curta. Isto certamente tem um impacto ambiental, que se faz notar principalmente pelo acúmulo em aterros sanitários. Medidas para minimizar estes problemas têm sido empregadas, destacando-se a reciclagem química e mecânica destes materiais. Na última década em especial, tanto a academia como o setor industrial investiram esforços na busca por alternativas de menor impacto ambiental para estes compósitos, dando origem a uma nova classe de compósitos denominados ecocompósitos ou biocompósitos ${ }^{[1,2]}$. Se por um lado estes materiais podem apresentar uma menor vida útil, eles devem apresentar propriedades térmicas e mecânicas semelhantes aos polímeros atualmente utilizados, associadas à possibilidade de biodegradação pós-consumo ${ }^{[2]}$.

As fibras vegetais ou lignocelulósicas constituem numa importante classe de materiais de reforço para matrizes poliméricas. Estas apresentam a estrutura de um compósito formado por lignina, que atua como matriz, e as fibrilas de celulose como reforço ${ }^{[1,2]}$. As vantagens das fibras vegetais sobre as fibras sintéticas são a baixa densidade, boas propriedades térmicas, biodegradabilidade, melhores propriedades mecânicas específicas, além de serem menos abrasivas aos equipamentos de processamento de polímeros ${ }^{[3]}$.

Geralmente, em compósitos a adesão entre as fases tem impacto significativo sobre as propriedades mecânicas ${ }^{[3,4]}$. Devido a este fato e a natureza química da matriz polimérica, muitas vezes faz-se necessário o tratamento físico ou químico da superfície das fibras lignocelulósicas. Estes tratamentos propiciam uma melhor fibrilação e dispersão das fibras pelas matrizes poliméricas, o aumento da rugosidade e alteração da polaridade superficial e a diminuição da adsorção de água ${ }^{[5]}$. Em geral, tratamentos químicos têm um impacto econômico considerável no preparo de compósitos, pelo uso de insumos químicos e pela geração de efluentes ${ }^{[6]}$. Fluidos supercríticos são utilizados na indústria química, incluindo áreas como a farmacêutica, de combustíveis, petroquímica e alimentícia, em processos, tais como: extrações, fracionamentos, separações e purificações ${ }^{[7,8,9]}$. Especificamente, tratamentos com fluidos supercríticos são aplicados a fibras lignocelulósicas objetivando a extração da lignina ${ }^{[7,8]}$, tendo como vantagem sobre os métodos convencionais de tratamento 
de fibras lignocelulósicas, tal como a mercerização, o fato de não gerar subprodutos ou rejeitos químicos. A extração de lignina de fibras lignocelulósicas provoca mudanças na rugosidade superficial, assim como a diminuição na adsorção de água. Esta última resulta principalmente do fato de que a lignina é um composto rico em hidroxilas, responsável pela sua hidroficilidade e afinidade com a água $^{[6,9]}$.

$\mathrm{Na}$ literatura têm sido reportadas as propriedades térmicas e mecânicas de compósitos poliméricos com fibras vegetais. Por exemplo, Takagi e cols. estudaram a condutividade térmica de biocompósitos de poli(acido láctico) (PLA) com fibras de bambu e observaram uma menor condutividade térmica para estes biocompósitos em comparação com a condutividade térmica de compósitos de PLA com fibra de vidro ou com fibra de carbono ${ }^{[10]}$. Kim e cols. estudaram compósitos de polipropileno modificado com anidrido maleico (agente de acomplamento) e fibras lignocelulósicas (cânhamo, sisal e linho). Tanto a condutividade térmica, como a resistência a tração são favorecidas pela presença do agente compatibilizante devido a uma melhoria na adesão interfacial ${ }^{[11]}$.

Santos e cols. estudaram compósitos de poliamida-6 e fibras curtas de Curauá submetidas a tratamento com plasma ou mercerizadas, visando melhorar a adesão interfacial. As melhores propriedades mecânicas a tração foram observadas para os compósitos com fibras tratadas com plasma ${ }^{[12]}$. Taha e Ziegmann estudaram as diferenças na adesão interfacial de fibras lignocelulósicas (linho e cânhamo) em matrizes biodegradáveis (acetato de celulose) e matrizes poliméricas não biodegradáveis (polietileno e polipropileno) ${ }^{[13]}$. Os resultados indicaram uma melhor adesão interfacial entre as fibras lignocelulósicas e o acetato de celulose, devido ao caráter hidrofílico de ambos componentes. Como consequência observou-se melhoras nas propriedades mecânicas a tração destes compósitos quando comparadas com os compósitos de fibras naturais e poliolefinas, as quais apresentam adesão fibra/matriz deficiente.

O objetivo deste trabalho foi desenvolver compósitos de acetato de celulose plastificado e fibras de Curauá tratadas e não tratadas com $\mathrm{CO}_{2}$ supercrítico. A opção por extrair lignina com $\mathrm{CO}_{2}$ supercrítico baseia-se no fato deste método representar um processo "quimicamente limpo", tal como descrito anteriormente. $\mathrm{O}$ acetato de celulose é um polímero biodegradável, amorfo e com alta resistência ao impacto quando plastificado ${ }^{[14]}$. As fibras de Curauá apresentam elevado modulo elástico e baixa densidade em comparação a outras fibras vegetais ${ }^{[15,16]}$. O uso dos plastificantes tem a finalidade de melhorar a processabilidade do acetato de celulose, assim como conferir flexibilidade ao polímero. Os ftalatos são plastificantes normalmente usados em matrizes polares, porém eles são tóxicos e não biodegradáveis ${ }^{[17]}$. Para garantir biocompósitos totalmente biodegradáveis é necessário utilizar plastificantes que tenham esta característica, tal como o citrato de trietila, que nos últimos anos tem sido testado em diferentes matrizes poliméricas biodegradáveis, preservando assim o caráter biodegradável ${ }^{[18]}$.

\section{Experimental}

Para o preparo dos biocompósitos foram utilizados o acetato de celulose livre de plastificantes e aditivos (Eastman Chemical Company, USA, 38,7\% de grupos acetila, Mn 50.000 g.mol ${ }^{-1}$ ) e o acetato de celulose contendo $30 \%$ em massa do plastificante ftalato de dioctila (DOP) e livre de outros aditivos comercial (Tenite acetate 105, Eastman Chemical Company, USA, 39,6\% de grupos acetila, Mn 30.000 g. $\mathrm{mol}^{-1}$ ). Citrato de trietila (TEC) (Across organics, USA) foi adicionado como plastificante ao acetato de celulose não plastificado na razão de $30 \%$ em massa. As fibras de Curauá (EMBRAPA-PA) foram previamente lavadas com água e secas ao ar e posteriormente moídas em um moinho de facas Rone - NFA1533 para a obtenção de fibras curtas com comprimento de 3 a $6 \mathrm{~mm}$.

Dióxido de carbono em condições supercríticas foi usado no tratamento das fibras. Cerca de $10 \mathrm{~g}$ de fibras de Curauá foram submetidas a extração com $\mathrm{CO}_{2}$ supercrítico a um fluxo de $8,75 \mathrm{~g} / \mathrm{min}$, por um tempo de 30 minutos a $180{ }^{\circ} \mathrm{C}$ e pressão de $16,5 \mathrm{MPa}^{[9]}$. Fibras de Curauá foram analisadas na região de infravermelho por refletância total atenuada (ATR) antes e após tratamento com $\mathrm{CO}_{2}$ supercrítico num equipamento Nicolet 380 FT-IR (Thermo scientific) na faixa de 650 a $4000 \mathrm{~cm}^{-1}$, resolução de $4 \mathrm{~cm}^{-1}$ e 64 acumulações. As fibras de Curauá tratadas e não tratadas também foram observadas com um microscópio ótico Nikon Eclipse 80i e fotografadas com uma câmara Nikon DS-Fi1. O diâmetro das fibras antes e após os tratamentos foi determinado com o auxílio do software NIS-Elements.

Os compósitos foram preparados usando acetato de celulose plastificado com DOP e TEC e fibras tratadas e não tratadas. O teor de plastificante e fibra foi mantido em $30 \%$ e $10 \%$ em massa, respectivamente (vide Tabela 1). Acetato de celulose e TEC foram pré-misturados por 24 horas antes da extrusão. Posteriormente acetato de celulose plastificado (com DOP ou TEC) e as fibras de Curaua foram alimentados em uma extrusora dupla rosca em escala de laboratório (DSM xplore $15 \mathrm{~cm}^{3}$ micro compounder). O processo de extrusão foi conduzido com três zonas de temperaturas (180, 190 e $200{ }^{\circ} \mathrm{C}$ ), velocidade da rosca na alimentação e no processo de 150 e $250 \mathrm{rpm}$, respectivamente, com um tempo de processamento de 3 minutos. Corpos de prova foram moldados por injeção usando a DSM explore $10 \mathrm{~cm}^{3}$, com uma temperatura no barril e no molde de 200 e $50{ }^{\circ} \mathrm{C}$, respectivamente. O tempo de injeção foi de 10 segundos a uma pressão de $0,8 \mathrm{MPa}$.

A condutividade térmica do acetato de celulose plastificado e seus compósitos foi calculada utilizando dados de capacidade calorífica determinados experimentalmente por calorimetria diferencial de varredura modulada (MDSC) no equipamento TA instruments 2910, segundo a norma ASTM E 1952. Para isso o equipamento foi calibrado com respeito a temperatura, capacidade calorífica $(\mathrm{Cp})$ e condutividade térmica $(\lambda)$ usando índio, safira e poliestireno sem aditivos (Aldrich), respectivamente. As amostras em forma de cilindros com alturas de 0,4 e $5 \mathrm{~mm}$, e com diâmetro

Tabela 1. Coeficiente de expansão térmica linear antes e apos a Tg, módulo elástico e resistência ao impacto para o acetato de celulose plastificado e seus compósitos com fibras de Curauá.

\begin{tabular}{|c|c|c|c|c|c|c|}
\hline \multirow{2}{*}{ Formulação } & \multirow{2}{*}{$\begin{array}{l}\text { Plastificante } \\
\qquad(30 \%)\end{array}$} & \multirow{2}{*}{$\begin{array}{l}\text { Fibra de Curauá } \\
\qquad(10 \%)\end{array}$} & \multicolumn{2}{|c|}{$\begin{array}{l}\text { Coeficiente de expansão térmica linear } \\
\left(\mu \mathrm{m} . \mathrm{mm}^{-1}{ }^{\circ} \mathrm{C}\right)\end{array}$} & \multirow{2}{*}{$\begin{array}{l}\text { Modulo elástico } \\
\text { (MPa) }\end{array}$} & \multirow{2}{*}{ 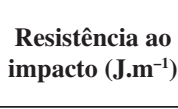 } \\
\hline & & & $\mathrm{T}<\mathrm{Tg}$ & $\mathrm{T}>\mathrm{Tg}$ & & \\
\hline CA30T & TEC & 0 & $166 \pm 9$ & $260 \pm 14$ & $532 \pm 28$ & $232 \pm 5$ \\
\hline CA30P & DOP & 0 & $219 \pm 12$ & $333 \pm 17$ & $494 \pm 17$ & $327 \pm 7$ \\
\hline CA30T10F & TEC & sem tratamento & $188 \pm 11$ & $419 \pm 22$ & $534 \pm 28$ & $60 \pm 4$ \\
\hline CA30P10F & DOP & sem tratamento & $254 \pm 14$ & $414 \pm 21$ & $540 \pm 20$ & $73 \pm 2$ \\
\hline $\mathrm{CA}_{30 \mathrm{P} 10 \mathrm{FCO}_{2}}$ & DOP & $\mathrm{CO}_{2}$ supercrítico & $203 \pm 11$ & $369 \pm 14$ & $502 \pm 17$ & $70 \pm 2$ \\
\hline
\end{tabular}


de $5 \mathrm{~mm}$, foram mantidos em diferentes temperaturas $(20,40,60$, 80 e $100{ }^{\circ} \mathrm{C}$ ) por 20 minutos, sob oscilação de temperatura com amplitude de $\pm 0,5{ }^{\circ} \mathrm{C}$ e período de 80 segundos, para o cálculo de $\mathrm{Cp}$ e de $\lambda$ em cada temperatura, de acordo com o descrito na referência ${ }^{[19]}$.

O coeficiente linear de expansão térmica foi calculado de dados obtidos em ensaios termomecânicos realizados no equipamento TMA2940 Thermomechanical Analyzer (TA Instruments). As análises foram realizadas segundo o programa: equilíbrio a $0{ }^{\circ} \mathrm{C}$, rampa de 0 até $120{ }^{\circ} \mathrm{C}$ a uma taxa de aquecimento de $5{ }^{\circ} \mathrm{C} / \mathrm{min}$. Foram utilizadas amostras na forma cilíndrica, com diâmetros de $5 \mathrm{~mm}$ e altura entre 5 e $7 \mathrm{~mm}$, segundo a norma ASTM E 831 . Uma calibração prévia foi realizada com um cilindro de alumínio de diâmetro $5 \mathrm{~mm}$ e altura de $8 \mathrm{~mm}$ segundo a norma ASTM E 2113.

O equipamento TGA 2950 foi utilizado para realizar estudos de estabilidade termo-oxidativa das fibras e dos compósitos, numa faixa de temperatura de 25 a $700{ }^{\circ} \mathrm{C}$, taxa de aquecimento de $5^{\circ} \mathrm{C} / \mathrm{min}$, sob atmosfera de ar sintético.

A análise dinâmico-mecânica foi conduzida em um equipamento Rheometrics Scientific DMTA V. As análises foram realizadas na faixa de $-50{ }^{\circ} \mathrm{C}$ a $250{ }^{\circ} \mathrm{C}$, a uma frequiência de $1 \mathrm{~Hz}$, amplitude de $0,01 \%$ e taxa de aquecimento de $2{ }^{\circ} \mathrm{C} / \mathrm{min}$. As dimensões das amostras foram de $6 \mathrm{~mm}$ de espessura, $12 \mathrm{~mm}$ de largura e $38 \mathrm{~mm}$ de comprimento.

Ensaios mecânicos de tração foram realizados usando uma máquina universal de ensaios (EMIC Universal Testing Machine DL2000) com uma célula de carga de 5000 N. Os corpos de prova foram acondicionados durante 48 horas a $25^{\circ} \mathrm{C}$ e $50 \%$ de umidade, segundo a norma ASTM D 638. Pelo menos oito corpos de prova de cada formulação foram ensaiados. Por outro lado, ensaios de resistência ao impacto modo Izod foram realizados em um aparelho EMIC tipo pêndulo, de acordo as especificações da norma ASTM

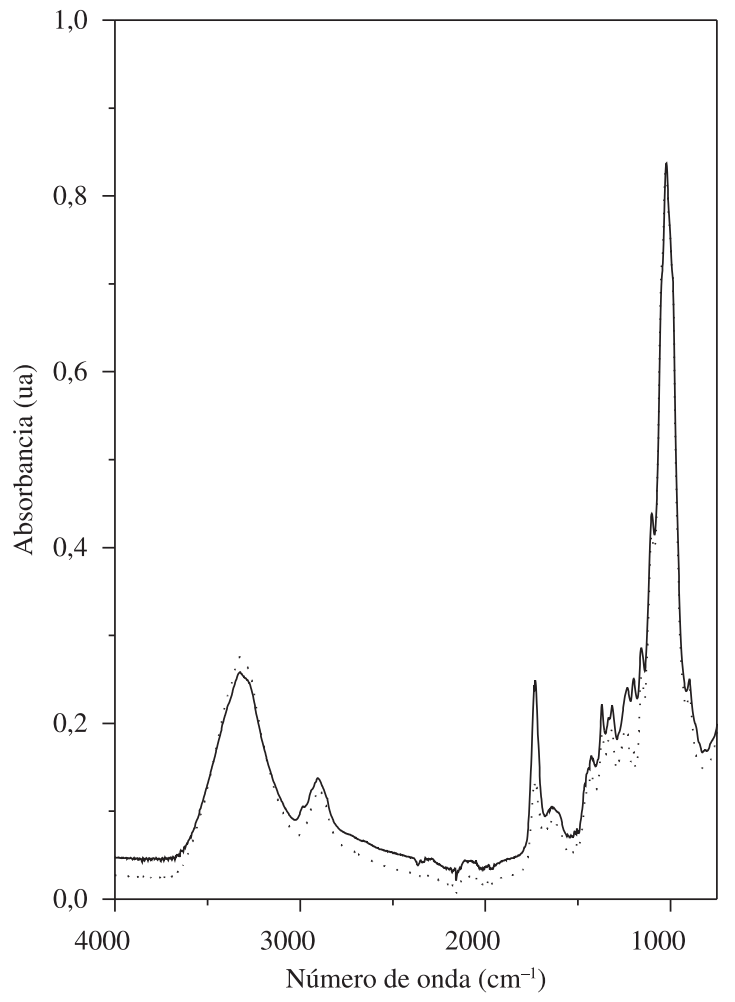

(a)
D 256. Pelo menos cinco corpos de prova de cada formulação de compósitos foram testados. As superfícies resultantes das fraturas dos ensaios de resistência ao impacto foram revestidas com carbono e ouro, para posterior análise em um microscópio JEOL JSM-6390 LV, operando a $20 \mathrm{kV}$.

\section{Resultados e Discussões}

$\mathrm{Na}$ Figura 1a são apresentados os espectros na região de infravermelho para as fibras de Curauá antes e após o tratamento com $\mathrm{CO}_{2}$ supercrítico. Os espectros apresentam basicamente o mesmo conjunto de absorções, porém, com diferenças nas intensidades relativas. A lignina por ser uma macromolécula aromática, apresenta absorções características de ligações $\mathrm{C}=\mathrm{C}$ em torno de $1622 \mathrm{~cm}^{-1}$. Uma banda centrada em $1260 \mathrm{~cm}^{-1}$ é atribuída aos grupos acetila da lignina ${ }^{[20]}$. Outras absorções nos espectros são observadas a $2950 \mathrm{~cm}^{-1}$, atribuída a estiramentos $\mathrm{C}-\mathrm{H}$ de grupos alifáticos, principalmente de segmentos da celulose, de hemicelulose e cera presentes na fibra; a $1030 \mathrm{~cm}^{-1}$, atribuída a estiramentos das ligações C-O-C presentes na celulose e hemicelulose e possivelmente lignina; em $3300 \mathrm{~cm}^{-1}$, referente a estiramento dos grupos hidroxila $(\mathrm{O}-\mathrm{H})$ da celulose principalmente; e em $1740 \mathrm{~cm}^{-1}$ a banda de estiramento dos grupos carbonilas $(\mathrm{C}=\mathrm{O})^{[20-23]}$.

A maioria das bandas observadas nos espectros podem ser atribuídas aos grupos químicos presentes nos diferentes componentes com exceção da banda para $\mathrm{C}=\mathrm{C}$ (aromático). Assim, a razão entre a altura da banda centrada em $1622 \mathrm{~cm}^{-1}\left(\mathrm{I}_{\mathrm{C}=\mathrm{C}}\right)$ e as bandas a $2950 \mathrm{~cm}^{-1}\left(\mathrm{I}_{\mathrm{C}-\mathrm{H}}\right)$, e a $1030 \mathrm{~cm}^{-1}\left(\mathrm{I}_{\mathrm{C}-\mathrm{O}-\mathrm{C}}\right)$, permite avaliar qualitativamente as principais modificações químicas das fibras tratadas com $\mathrm{CO}_{2}$ supercrítico. Para as fibras não tratadas é possível observar razões $\mathrm{I}_{\mathrm{C}-\mathrm{H}} / \mathrm{I}_{\mathrm{C}=\mathrm{C}}$ de 1,2 e $\mathrm{I}_{\mathrm{C}-\mathrm{O}-\mathrm{C}} / \mathrm{I}_{\mathrm{C}=\mathrm{C}}$ de 8,2 . Já para as fibras

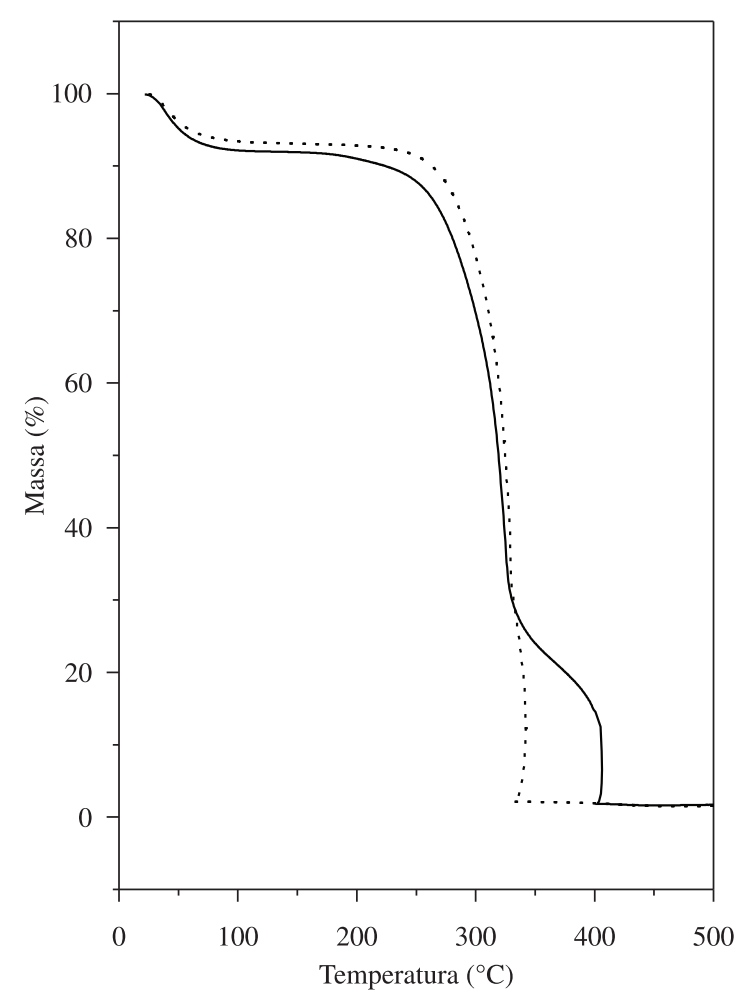

(b)

Figura 1. a) Espectro vibracional na região de infravermelho para as fibras de Curauá, resolução $4 \mathrm{~cm}^{-1} ; 64$ acumulações. b) Curvas termogravimétricas das fibras de Curauá, taxa de aquecimento de $5{ }^{\circ} \mathrm{C} / \mathrm{min}$; atmosfera oxidante. (-) Não tratadas e (‥) Tratadas com $\mathrm{CO}_{2}$ supercrítico. 
tratadas estas razões são de 1,4 e 10,2, respectivamente. O aumento nas intensidades relativas das bandas para $\mathrm{C}-\mathrm{H}$ e $\mathrm{C}-\mathrm{O}-\mathrm{C}$ reflete a diminuição dos grupos $\mathrm{C}=\mathrm{C}$ (associados a lignina) nas fibras tratadas com $\mathrm{CO}_{2}$ supercrítico com respeito às fibras não tratadas. Estes resultados indicam que a lignina foi parcialmente extraída pelo $\mathrm{CO}_{2}$ supercrítico. Comportamento similar foi reportado por Ouajai e Shanks para fibras de cânhamo após tratamento com soda ${ }^{[23]}$.

Fibras de Curauá tratadas e não tratadas foram analisadas por microscopia óptica para determinar os diâmetros das mesmas. Fibras não tratadas apresentaram diâmetro médio de $72 \pm 5 \mu \mathrm{m}$. As fibras tratadas com $\mathrm{CO}_{2}$ supercrítico apresentaram diâmetro médio de $10 \pm 2 \mu \mathrm{m}$. Esta diminuição no diâmetro das fibras após tratamento com fluidos supercríticos, pode ser associada tanto à deslignificação ${ }^{[1,2,5]}$, quanto às elevadas pressões a que são submetidas às fibras, que favorece a fibrilação.

Na Figura 1b são apresentadas as curvas termogravimétricas em atmosfera oxidante para as fibras tratadas e não tratadas. É possível observar que as fibras não tratadas apresentam 3 estágios de degradação termo-oxidativa. O primeiro estágio ocorre a temperaturas inferiores a $100{ }^{\circ} \mathrm{C}$ sendo atribuído a perda de compostos voláteis, como a umidade. O segundo estágio em torno de $300{ }^{\circ} \mathrm{C}$ é associado à ruptura das cadeias de celulose e hemicelulose. Por último, aproximadamente a $400{ }^{\circ} \mathrm{C}$ é observado o terceiro estágio de perda de massa devido à quebra da estrutura da lignina e formação de volateis, como foi reportado na literatura ${ }^{[15,16]}$. A curva termogravimétrica em atmosfera oxidante para as fibras tratadas apresenta só os dois primeiros estágios, mostrando que a extração da lignina foi bem sucedida.

A Figura 2 apresenta as micrografias obtidas por microscopia eletrônica de varredura para os compósitos plastificados com DOP e TEC. Para todos os compósitos constata-se a distribuição uniforme de fibrilas (Figuras 2c e 2d). O diâmetro das fibrilas é da ordem de $5 \mu \mathrm{m}$ para as fibras tratadas e não tratadas [diâmetro médio da fibra não tratada é de $72 \pm 5$ ) $\mu \mathrm{m}$ ], não se observando diferenças entre as mesmas com respeito a rugosidade superficial (Figuras 2a e 2b). A análise das micrografias revela regiões em que há nitidamente espaços vazios entre as fibrilas e a matriz, enquanto em outras regiões estes espaços não são observados. Portanto, a simples análise das fraturas dos compósitos não permite concluir se a extração das fibras de Curauá com $\mathrm{CO}_{2}$ supercrítico resultou em alterações nas propriedades interfaciais dos biocompósitos.

As curvas termogravimétricas em atmosfera oxidante para o acetato de celulose plastificado e seus compósitos com fibras de Curauá são apresentadas na Figura 3. O perfil das curvas

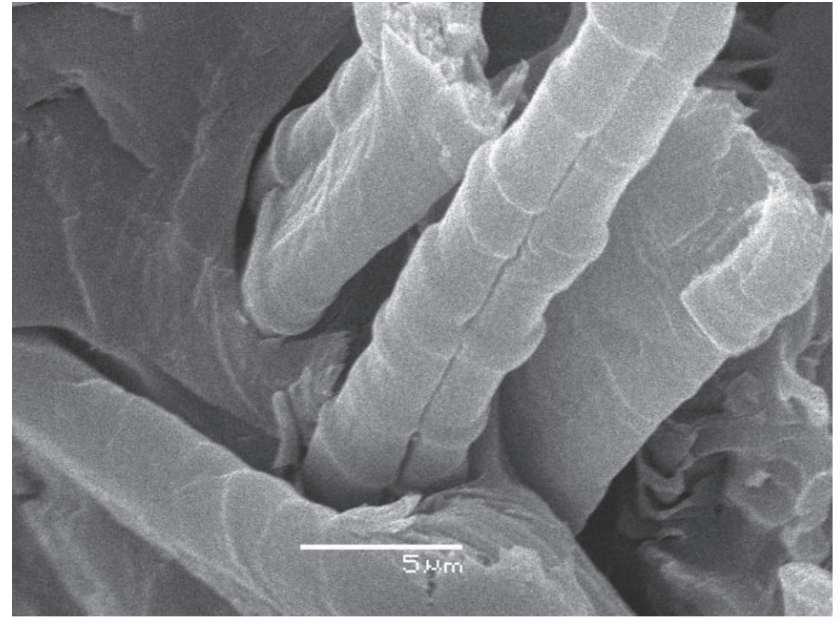

(a)

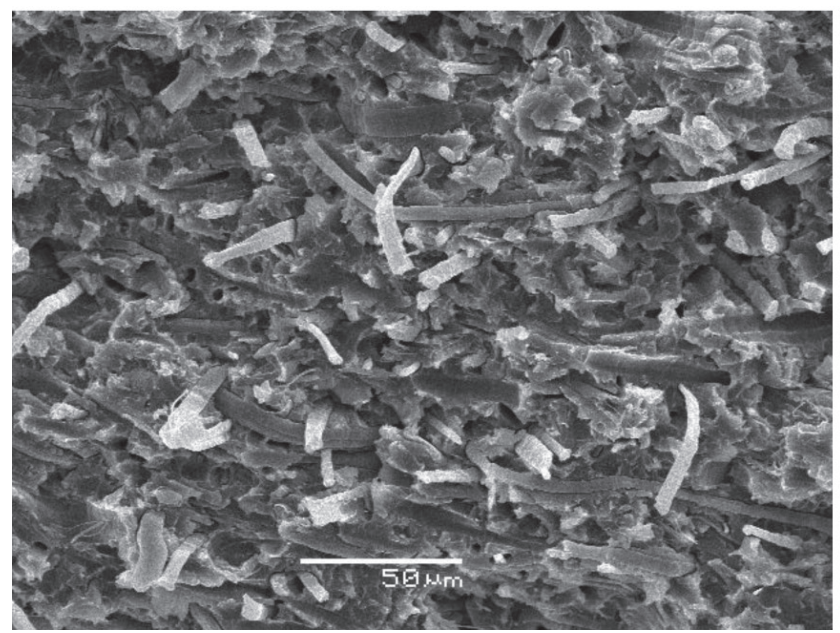

(c)

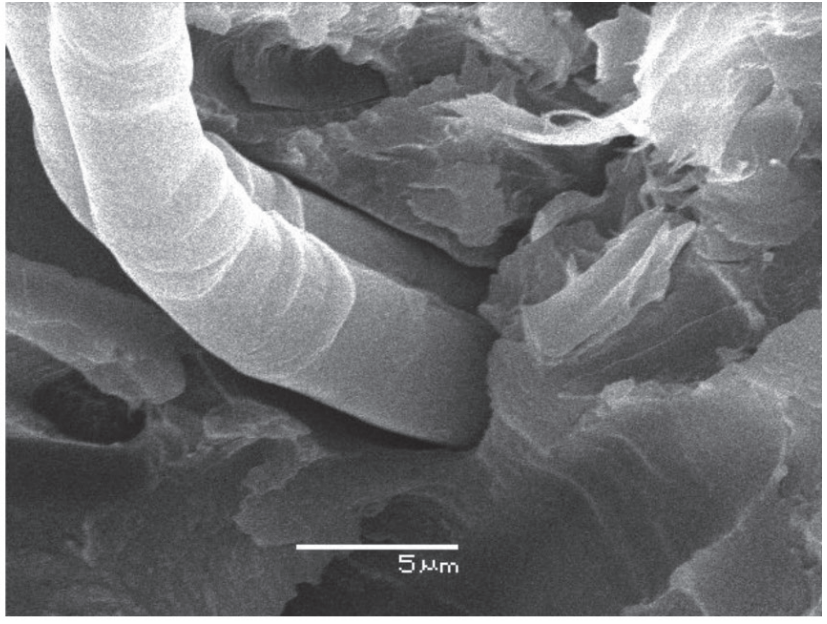

(b)

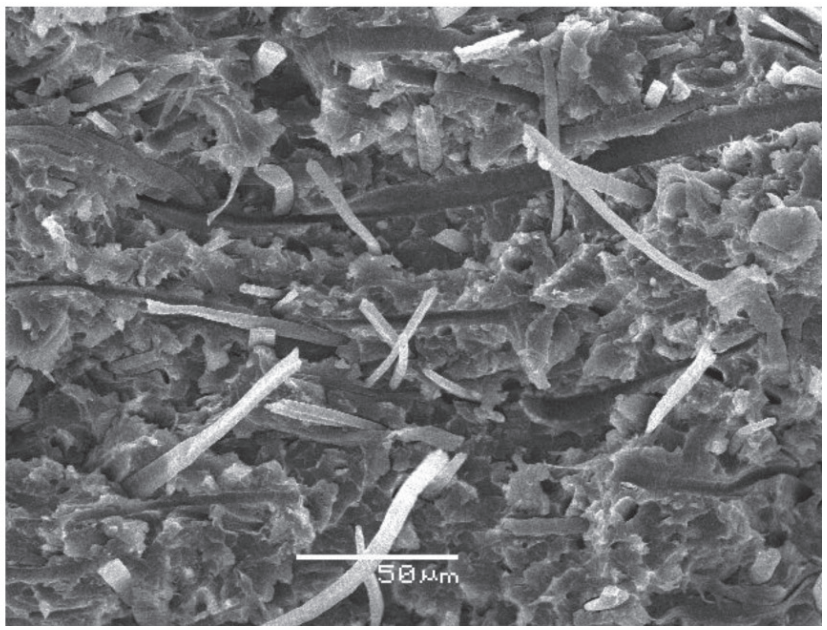

(d)

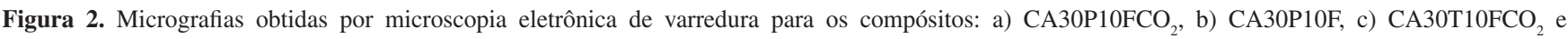
d) CA30T10F. 
termogravimétricas para o acetato de celulose plastificado e os compósitos são similares, mostrando quatro estágios de perda de massa. $\mathrm{O}$ primeiro, ao redor de $100{ }^{\circ} \mathrm{C}$, é atribuído à volatilização de umidade, sorvida tanto pelas fibras como pelo acetato de celulose ${ }^{[16]}$. Entre $250{ }^{\circ} \mathrm{C}$ e $300{ }^{\circ} \mathrm{C}$ ocorre uma segunda perda de massa, associada à volatilização dos plastificantes ${ }^{[23]}$. A liberação dos grupos acetila das cadeias de acetato de celulose, assim como a ruptura das cadeias poliméricas, tanto do acetato de celulose como dos produtos celulósicos das fibras, acontece a $350{ }^{\circ} \mathrm{C}^{[22,24]}$. Acima de $380{ }^{\circ} \mathrm{C}$ ocorre a oxidação dos resíduos ricos em carbono ${ }^{[25]}$. É possível observar uma menor temperatura de oxidação dos resíduos para os compósitos em relação ao acetato de celulose plastificado, devido à presença de grupos oxidantes na lignina como as hidroxilas, o que acelera a oxidação dos produtos finais nos biocompósitos.

A Figura 4a apresenta as curvas de módulo de armazenamento $\left(\mathrm{E}^{\prime} \times \mathrm{T}\right)$ para o acetato de celulose plastificado e para os compósitos com fibras de Curauá. As curvas do módulo de armazenamento para o acetato de celulose plastificado com TEC e seus compósitos apresentam uma queda de três ordens de grandeza em $80{ }^{\circ} \mathrm{C}$, enquanto as formulações plastificadas com DOP apresentam uma queda de magnitude similar em $75{ }^{\circ} \mathrm{C}$, atribuídas à transição vítrea do acetato de celulose plastificado. A diferença na temperatura de transição vítrea $(\mathrm{Tg})$ indica a eficiência dos plastificantes, sendo o DOP um plastificante ligeiramente mais eficiente que o TEC para o acetato de celulose. Para os compósitos observa-se uma atenuação na queda do módulo de armazenamento em comparação ao acetato de celulose plastificado a temperaturas acima da transição vítrea, devido ao efeito de reforço conferido pelas fibras de Curauá.

As curvas de módulo de perda em função da temperatura $(\mathrm{E}$ ” $\times \mathrm{T})$ para o acetato de celulose plastificado e seus compósitos são apresentadas na Figura 4b. Videki et al. ${ }^{[26]}$ relataram para o acetato de celulose três transições: a transição vítrea a $85^{\circ} \mathrm{C}$, a transição $\beta \mathrm{a}-5^{\circ} \mathrm{C}$, atribuída às relaxações dos anéis de glicose e a transição $\gamma \mathrm{a}-85^{\circ} \mathrm{C}$ associados aos grupos hidroxila que interagem com as moléculas de água sorvidas. As curvas do módulo de perda em função da temperatura para o acetato de celulose e seus compósitos com fibras de Curauá apresentam apenas a transição vítrea e a

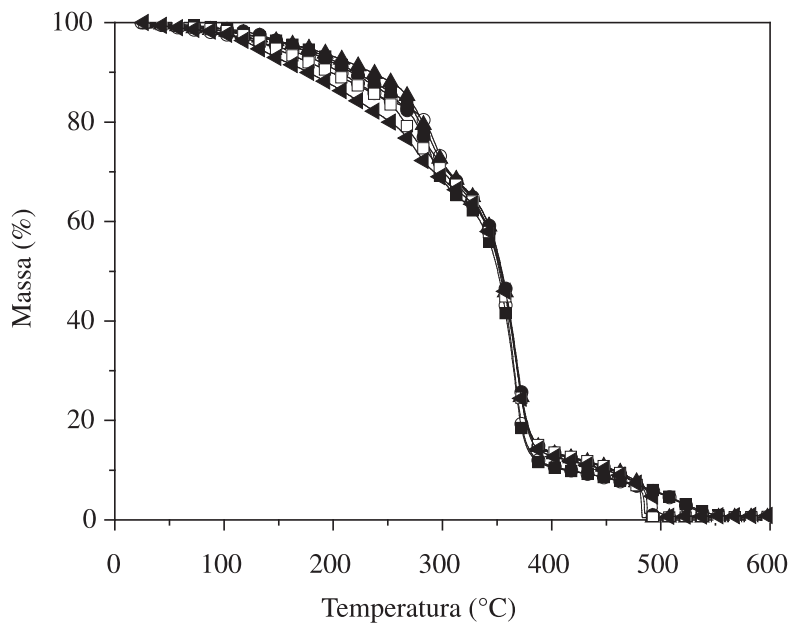

(a) transição secundária $(\beta)$, na faixa de temperatura analisada. Para os materiais plastificados com TEC, observa-se um primeiro pico a $-25^{\circ} \mathrm{C}$ atribuído à transição secundária, e um segundo pico a $80^{\circ} \mathrm{C}$ associado à transição vítrea do acetato de celulose plastificado. Enquanto para os materiais plastificados com DOP estas relaxações se apresentam a $-38{ }^{\circ} \mathrm{C}$ e $75{ }^{\circ} \mathrm{C}$, respectivamente. Este resultado deve-se ao fato do volume livre do acetato de celulose plastificado com DOP ser maior, resultando em menor Tg comparativamente à Tg para o acetato de celulose plastificado com TEC. Esta diferença de volume livre reflete o volume molar dos plastificantes. Enquanto o DOP apresenta um volume molar de $396,5 \mathrm{~cm}^{3} \cdot \mathrm{mol}^{-1}$, o volume molar do TEC é de $243,5 \mathrm{~cm}^{3} \cdot \mathrm{mol}^{-1[27]}$. As temperaturas referentes a transição vítrea e a transição secundária $(\beta)$ não são afetadas pela presença das fibrilas. Porém, é possível observar um alargamento das curvas de módulo de perda em função da temperatura para os compósitos na região de transição vítrea, que poderia refletir um gradiente de composição ou um gradiente de mobilidade molecular. É possível que as cadeias de acetato de celulose próximas das fibrilas tenham sua mobilidade afetada.

A capacidade calorífica a diferentes temperaturas para o acetato de celulose e seus compósitos encontra-se na Tabela 2. O acetato de celulose plastificado com DOP apresenta valores superiores da capacidade calorífica em comparação aos valores observados para o acetato de celulose plastificado com TEC em toda a faixa de temperatura avaliada. A capacidade calorífica é uma função dos graus de liberdade que, no caso de macromoléculas a temperaturas inferiores e próximas a transição vítrea, se restringe a graus de liberdade rotacionais associados a grupos pendentes de cadeias ou a poucos segmentos da cadeia principal e vibracionais. Os primeiros estão relacionados às relaxações secundárias de macromoléculas ${ }^{[28]}$. O fato de DOP conferir maior capacidade calorífica ao acetato de celulose plastificado indica um aumento nos graus de liberdade das cadeias poliméricas, e com isto uma maior flexibilidade do material.

Os compósitos apresentam capacidade calorífica maior em comparação com o seu correspondente acetato de celulose plastificado. Dentre os compósitos, aqueles que contêm fibras tratadas com $\mathrm{CO}_{2}$ apresentam maior capacidade calorífica. Este comportamento pode decorrer da extração de lignina, que atua como

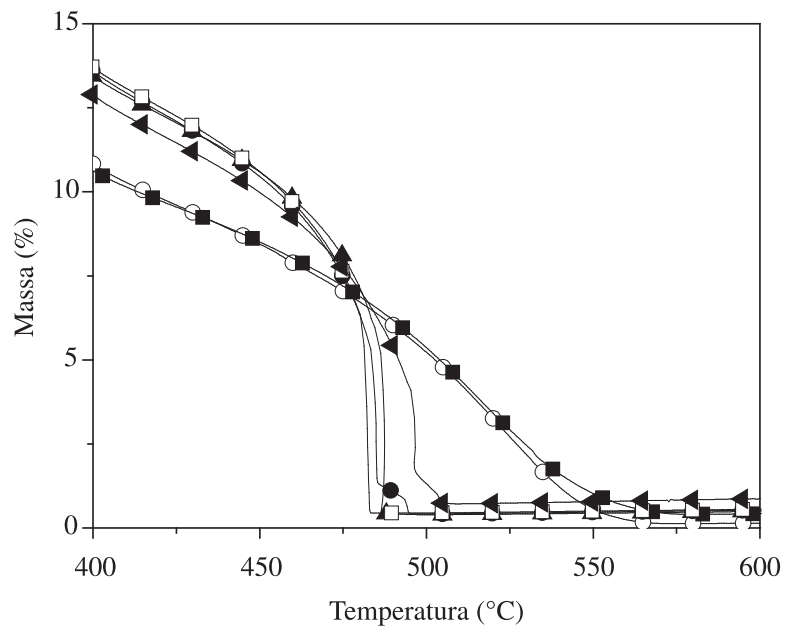

(b)

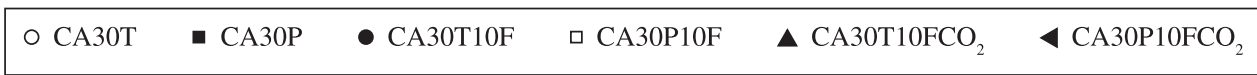

Figura 3. a) Curvas termogravimétricas para o acetato de celulose plastificado e seus compósitos com fibras de Curauá: (o) CA30T, (匹) CA30P, (•) CA30T10F, (口) CA30P10F, ( $\mathbf{\Delta}$ ) CA30T10FCO e ( \) $\mathrm{CA} 30 \mathrm{P} 10 \mathrm{FCO}_{2}$; b) ampliação das curvas na região a 400 a $600{ }^{\circ} \mathrm{C}$. Taxa de aquecimento de $5{ }^{\circ} \mathrm{C} / \mathrm{min}$; atmosfera oxidante. 
um adesivo ou matriz para as fibrilas. Estes resultados indicam que a retirada de lignina contribui para o aumento da flexibilidade das fibrilas e, por tanto, para o aumento da capacidade calorífica dos compósitos.

Na Tabela 3 são apresentados os valores de condutividade térmica para o acetato de celulose plastificado e seus compósitos. Para ambos, é possível observar um aumento na condutividade térmica com o aumento da temperatura até a transição vítrea. A temperaturas acima da Tg o acetato de celulose e seus compósitos

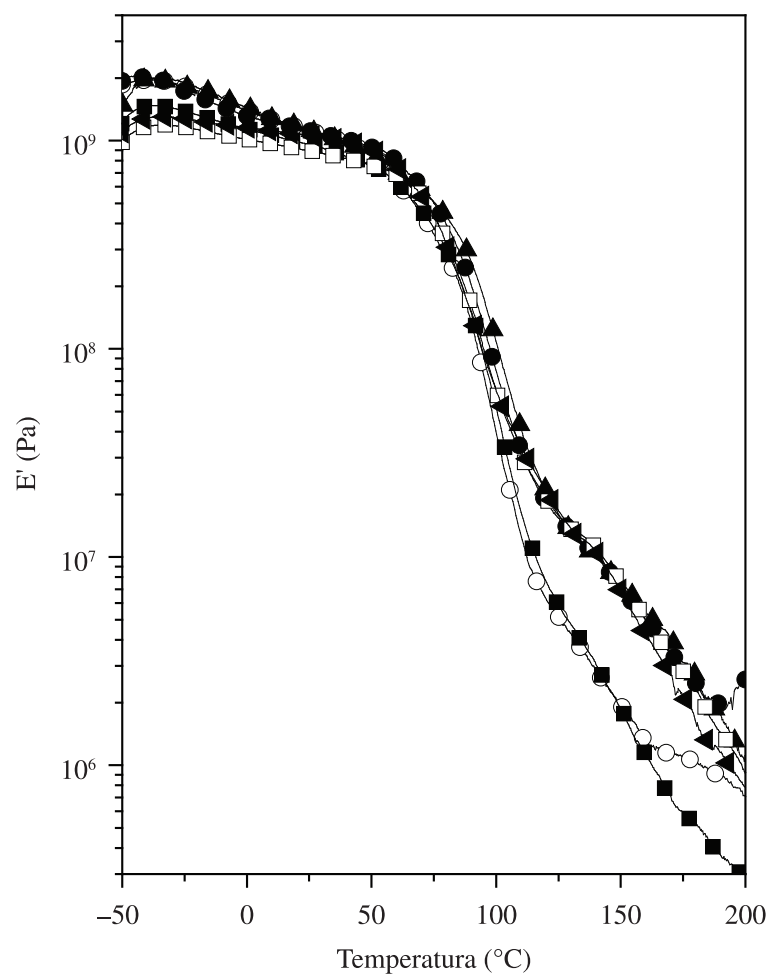

(a) apresentam uma queda na condutividade térmica. Em polímeros amorfos isolantes, como o acetato de celulose, a temperaturas abaixo da $\mathrm{Tg}$ o mecanismo de transferência de calor é baseado no modelo de rede de fônon - fracton. A aplicação deste modelo a polímeros amorfos pressupõe uma ordem de médio alcance ${ }^{[29]}$. Já a temperaturas acima da $\mathrm{Tg}$, a elevada mobilidade das cadeias poliméricas e o espaçamento entre as cadeias interferem negativamente na transferência da energia térmica, observando-se uma queda da condutividade térmica. Este comportamento é

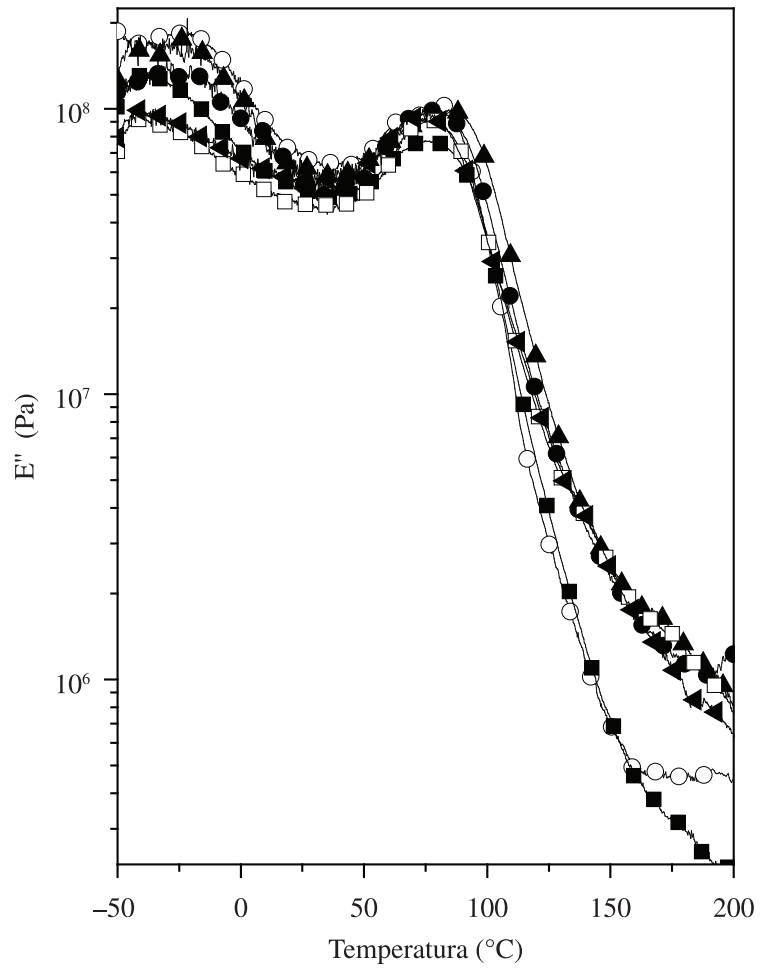

(b)

\section{O CA30T - CA30P $\bullet \mathrm{CA} 30 \mathrm{~T} 10 \mathrm{~F} \quad \square \mathrm{CA} 30 \mathrm{P} 10 \mathrm{~F} \quad \boldsymbol{\mathrm { CA }} 30 \mathrm{~T} 10 \mathrm{FCO}_{2} \quad 4 \mathrm{CA}_{30 \mathrm{P} 10 \mathrm{FCO}}$}

Figura 4. Módulo de armazenamento (a) e de perda (b) em função da temperatura de acetato de celulose e seus compósitos com fibra de Curauá: (O) CA30T,

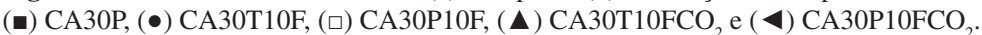

Tabela 2. Capacidade calorífica a diferentes temperaturas para o acetato de celulose plastificado e seus compósitos com fibra de Curauá.

\begin{tabular}{lccccc}
\hline \multicolumn{1}{c}{$\mathbf{C} \mathbf{p}\left(\mathbf{J}_{\mathbf{g}} \mathbf{g}^{\mathbf{1}} \cdot \mathbf{K}^{-\mathbf{1}}\right)$} & $\mathbf{2 0}{ }^{\circ} \mathbf{C}$ & $\mathbf{4 0}{ }^{\circ} \mathbf{C}$ & $\mathbf{6 0}{ }^{\circ} \mathbf{C}$ & $\mathbf{8 0}{ }^{\circ} \mathbf{C}$ & $\mathbf{1 0 0}{ }^{\circ} \mathbf{C}$ \\
\hline $\mathrm{CA30T}$ & $1,092 \pm 0,004$ & $1,158 \pm 0,003$ & $1,227 \pm 0,003$ & $1,308 \pm 0,008$ & $1,430 \pm 0,002$ \\
$\mathrm{CA30P}$ & $1,299 \pm 0,005$ & $1,290 \pm 0,003$ & $1,384 \pm 0,002$ & $1,472 \pm 0,001$ & $1,628 \pm 0,001$ \\
$\mathrm{CA30T10F}$ & $1,578 \pm 0,003$ & $1,678 \pm 0,003$ & $1,790 \pm 0,004$ & $1,902 \pm 0,004$ & $2,029 \pm 0,004$ \\
$\mathrm{CA30P10F}$ & $1,423 \pm 0,002$ & $1,526 \pm 0,003$ & $1,617 \pm 0,003$ & $1,729 \pm 0,003$ & $1,904 \pm 0,005$ \\
$\mathrm{CA30T10FCO}$ & $1,608 \pm 0,002$ & $1,724 \pm 0,006$ & $1,832 \pm 0,004$ & $1,953 \pm 0,003$ & $2,113 \pm 0,003$ \\
$\mathrm{CA30P10FCO}$ & $1,775 \pm 0,005$ & $1,886 \pm 0,001$ & $1,995 \pm 0,006$ & $2,065 \pm 0,005$ & $2,174 \pm 0,004$ \\
\hline
\end{tabular}

Tabela 3. Condutividade térmica a diferentes temperaturas para o acetato de celulose plastificado e seus compósitos com fibra de Curauá.

\begin{tabular}{lccccc}
\hline \multicolumn{1}{c}{$\boldsymbol{\lambda}\left(\mathbf{W} \cdot \mathbf{m}^{-1} \cdot \mathbf{K}^{-1}\right)$} & $\mathbf{2 0}{ }^{\circ} \mathbf{C}$ & $\mathbf{4 0}{ }^{\circ} \mathbf{C}$ & $\mathbf{6 0}{ }^{\circ} \mathbf{C}$ & $\mathbf{8 0}{ }^{\circ} \mathbf{C}$ & $\mathbf{1 0 0}{ }^{\circ} \mathbf{C}$ \\
\hline $\mathrm{CA} 30 \mathrm{~T}$ & $0,142 \pm 0,005$ & $0,146 \pm 0,007$ & $0,158 \pm 0,001$ & $0,159 \pm 0,005$ & $0,119 \pm 0,003$ \\
$\mathrm{CA30P}$ & $0,162 \pm 0,004$ & $0,165 \pm 0,002$ & $0,167 \pm 0,002$ & $0,159 \pm 0,008$ & $0,116 \pm 0,007$ \\
$\mathrm{CA30T10F}$ & $0,133 \pm 0,002$ & $0,137 \pm 0,004$ & $0,134 \pm 0,004$ & $0,134 \pm 0,006$ & $0,124 \pm 0,004$ \\
$\mathrm{CA30P10F}$ & $0,142 \pm 0,005$ & $0,145 \pm 0,005$ & $0,147 \pm 0,005$ & $0,145 \pm 0,002$ & $0,122 \pm 0,002$ \\
$\mathrm{CA30T10FCO}$ & $0,129 \pm 0,006$ & $0,131 \pm 0,008$ & $0,122 \pm 0,007$ & $0,120 \pm 0,005$ & $0,102 \pm 0,003$ \\
$\mathrm{CA30P10FCO}$ & $0,095 \pm 0,001$ & $0,098 \pm 0,009$ & $0,100 \pm 0,002$ & $0,104 \pm 0,007$ & $0,088 \pm 0,002$ \\
\hline
\end{tabular}


reportado por Dashora e Gupta, para polímeros amorfos não condutores como o poli(cloreto de vinila) e o poliestireno ${ }^{[30]}$.

Os dados da Tabela 3 mostram que o acetato de celulose plastificado com DOP a temperaturas inferiores abaixo a $\mathrm{Tg}$ apresenta condutividade térmica maior em comparação com o acetato de celulose plastificado com TEC. A introdução das fibras de Curauá tratadas ou não no acetato de celulose plastificado acarreta em diminuição da condutividade térmica em toda a faixa de temperatura, uma vez que uma segunda fase pode atuar como barreira para a transferência de energia. Para os compósitos com fibras tratadas a queda na condutividade térmica é observada em toda a faixa de temperatura avaliada em relação às outras formulações. Os biocompósitos de acetato de celulose e fibras curtas de Curauá são materiais densos e mesmo assim apresentam valores de condutividade térmica de mesma ordem de grandeza que materiais porosos tradicionalmente utilizados como isolantes térmicos, como, as espumas de poliuretanas $\left(0,02-0,04 \mathrm{~W} \cdot \mathrm{m}^{-1} \cdot \mathrm{K}^{-1}\right)$ e de poliestirenos $\left(0,03-0,06 \mathrm{~W} \cdot \mathrm{m}^{-1} \cdot \mathrm{K}^{-1}\right)^{[1]}$.

Na Tabela 1 são apresentados os valores dos coeficientes de expansão térmica linear $(\alpha)$ para o acetato de celulose plastificado e seus compósitos em função da temperatura. Abaixo da $\mathrm{Tg}$, é observado um maior valor de $\alpha$ para o acetato de celulose plastificado com DOP e seus compósitos, devido ao maior volume livre do polímero plastificado com DOP em comparação com formulações com TEC. Acima da Tg, há o aumento de $\alpha$ devido ao aumento dos graus de liberdade das cadeias poliméricas. Mahonty e cols. determinaram o coeficiente de expansão térmica linear do acetato de celulose plastificado com diferentes teores de TEC ${ }^{[31]}$. Para o acetato de celulose contendo $30 \%$ de TEC o coeficiente de expansão térmica antes da $\mathrm{Tg}\left(80{ }^{\circ} \mathrm{C}\right)$ é de $160 \mu \mathrm{m} \cdot \mathrm{mm}^{-1} \cdot \mathrm{K}^{-1}$, valor próximo ao obtido neste trabalho $\left[(166 \pm 9) \mu \mathrm{m} \cdot \mathrm{mm}^{-1} \cdot \mathrm{K}^{-1}\right]$. Porém, os compósitos com fibras tratadas com fluido supercrítico apresentam um $\alpha$ menor que aqueles com fibras não tratadas. A análise termogravimétrica das fibras (Figura 1b) mostrou que as fibras tratadas sorvem menos água. A presença de água por sua vez, influencia a estabilidade dimensional, o que poderia explicar os menores valores de $\alpha$ para compósitos contendo fibras menos hidrofílicas. Este comportamento também foi reportado por Fangueiro e Mukhopadhyay ${ }^{[5]}$.

As propriedades mecânicas para o acetato de celulose plastificado e para seus compósitos com fibras de Curauá são apresentados na Tabela 1. O DOP confirma ser um melhor plastificante para acetato de celulose, pois a resistência ao impacto é maior em comparação ao valor observado para o acetato de celulose plastificado com TEC. Para os compósitos, a presença de fibras de Curauá gera um discreto aumento no módulo de elasticidade (10\%) e uma perda significativa da resistência ao impacto (340\%). O discreto aumento no módulo elástico poderia estar associado à perda da estrutura das fibras em decorrência da fibrilação, assim como a diminuição da razão de aspecto como consequência do processamento tal como reportado por Le Baillif e Oskman ${ }^{[32]}$. No caso das fibras tratadas ainda deve ser considerada a extração da lignina, que interfere na estrutura das fibras. E, embora a análise morfológica da superfície de fraturas dos biocompósitos não tenha sido conclusiva no que diz respeito a adesão interfacial, as propriedades mecânicas sugerem que a adesão matrizfibra é deficitária, independentemente do tratamento das fibras.

Enquanto o acetato de celulose plastificado com DOP se apresenta como um material mais tenaz que o acetato de celulose plastificado com TEC, para os compósitos plastificados com DOP observa-se uma queda mais acentuada na resistência ao impacto, da ordem de $340 \%$, contra $280 \%$ para os compósitos plastificados com TEC, em comparação com os valores observados para os respectivos acetatos de celulose plastificados.

\section{Conclusões}

O tratamento das fibras de Curauá resultaram em extração parcial de lignina e fibrilação. Este material tratado, assim com as fibras não tratadas ao serem incorporadas a matriz de acetato de celulose plastificado resultou em compósitos nos quais as fibrilas estão distribuídas uniformemente. Os biocompósitos de acetato de celulose e fibras curtas de Curauá tratadas com fluidos supercríticos apresentaram maior capacidade calorífica, menor condutividade térmica e maior coeficiente de expansão térmica, em comparação aos compósitos com fibras não tratadas. Este comportamento é atribuído à extração de lignina das fibras, a fibrilação e a distribuição uniforme das fibrilas pela matriz polimérica. $\mathrm{O}$ aumento discreto do módulo de Young, e a queda significativa da resistência ao impacto apresentado pelos compósitos em relação ao acetato de celulose plastificado deve-se a fibrilação e diminuição de razão de aspecto das fibras de Curauá, durante o processamento de extrusão. A presença das fibras de Curauá tratadas e não tratadas não modifica o comportamento termo-oxidativo do acetato de celulose plastificado.

DOP se apresentou como um plastificante mais eficiente em comparação ao TEC devido ao maior volume livre conferido ao compósito plastificado com DOP. Por outro lado, as formulações plastificadas com TEC apresentaram menor condutividade térmica e menor coeficiente de expansão térmica em relação às formulações com DOP, fato este relacionado ao menor volume livre das primeiras formulações. Os biocompósitos contendo fibras de Curauá tratados ou não com $\mathrm{CO}_{2}$ supercrítico apresentam potencial de uso como isolantes térmicos. No caso dos biocompósitos plastificados com TEC tem-se ainda a possibilidade destes serem biodegradáveis.

\section{Agradecimentos}

Chávez M. agradece a CAPES pela bolsa de doutorado - programa PEC-PG. Os autores agradecem a FAPESP (2010/17804-7 e 2010/2098-0) e a PETROBRAS pelo apoio financeiro e à EMBRAPA-PA pelo fornecimento das fibras de Curauá.

\section{Referências Bibliográficas}

1. Mahonty, A. K.; Misra, M. \& Hinrichsen, G. - Macromol. Mater. Eng., 276-277, p.1 (2000).

2. John, M. J. \& Thomas, S. - Carbohydr. Polym., 71, p.341 (2008).

3. Bledzki, A. K.; Reihmane, S. \& Gassan J. - J. Appl. Polym. Sci., 59, p.1329 (1996). http://dx.doi.org/10.1002/(SICI)1097-4628(19960222) 59:8<1329::AID-APP17>3.0.CO;2-0

4. Aluigi, A.; Vineis, C.; Ceria, A. \& Tonin C. - Compos. Part A: Appl. Sci. Manufact., 39, p.126 (2008). http://dx.doi.org/10.1016/j. compositesa.2007.08.022

5. Fangueiro, R. \& Mukhopadhyay, S. - J. Therm. Comp. Mater., 22, p.135 (2009). http://dx.doi.org/10.1177/0892705708091860

6. Panigrahi, S.; Li, X. \& Tabil L. G. - J. Polym. Environ., 15, p.25 (2007). http://dx.doi.org/10.1007/s10924-006-0042-3

7. Kiran, E. \& Balkan, H. - J. Supercritical. Fluids, 7, p.75 (1994). http:// dx.doi.org/10.1016/0896-8446(94)90043-4

8. Li, L. \& Kiran, E. - Ind. Eng. Chem. Res., 27, p.1301 (1988). http:// dx.doi.org/10.1021/ie00079a035

9. Pasquine, D.; Pimenta, M. T.; Ferreira, L. H. \& Curvelo, A. S. A. - J. Supercritical Fluids, 36, p.31 (2005). http://dx.doi.org/10.1016/j. supflu.2005.03.004

10. Takagi,H.; Kako, S.; Kusano, K. \& Ousaka,A. -Adv. Compos. Mater., 16, p. 377 (2007). http://dx.doi.org/10.1163/156855107782325186

11. Kim, S. W.; Lee, S. H.; Kang, J. S. \& Kang, K. H. - Inter. J. Thermophys., 27, p. 1873 (2006). http://dx.doi.org/10.1007/ s10765-006-0128-0 
12. Santos, P. A.; Spinacé, M. A. S.; Fermoselli, K. K. G. \& De Paoli, M. A. - Polímeros, 19, p.31 (2009).

13. Taha, I. \& Ziegmann, G. - J. Compos. Mater., 40, p.1933 (2006). http:// dx.doi.org/10.1177/0021998306061304

14. Ouajai, S. \& Shanks, R. A. - Macromol. Mater Eng., 294, p.213 (2009). http://dx.doi.org/10.1002/mame.200800266

15. D’Almeida, J. R. M.; Aquino, R. C. M. P. \& Monteiro, S. N. - Compos. Part A, Appl. Sci. Manuf., 37, p.1473 (2006).

16. Spinacé, M. A. S.; Lambert, C. S.; Fermoselli, K. K. G. \& De Paoli, M. A. - Carbohydr. Polym., 77, p.47 (2009).

17. Persico, P.; Ambrogi, V.; Acierno, D. \& Carfagna, C. - J. Vinyl. Addit. Technol., 15, p.139 (2009). http://dx.doi.org/10.1002/vnl.20187

18. Wong, S.; Shanks, R. A. \& Hodzic A. - Polym. Eng. Sci., 43, p.1566 (2007). http://dx.doi.org/10.1002/pen.10132

19. Lopes, C. M. A.; Felisberti, M. I. - Polym. Test., 23, p. 637 (2004). http://dx.doi.org/10.1016/j.polymertesting.2004.01.013

20. Silva, R. V. \& Aquino, E. M. F. - J. Reinforc. Plast. Compos., 27, p.103 (2008). http://dx.doi.org/10.1177/07316844080270010301

21. Phuong, N. T.; Solloguob, C. \& Guinault, A. - J. Reinforc. Plast. Compos., 29/21, p.3244 (2010).

22. Tomczak, F.; Satyanarayana, K. G. \& Sydenstricker, T. H. D. - Compos. Part A: Appl. Sci. Manufact., 38, p.2227 (2007). http://dx.doi. org/10.1016/j.compositesa.2007.06.005
23. Ouajai, S. \& Shanks, R. A. - Compos. Sci. Technol., 69, p.2119 (2009). http://dx.doi.org/10.1016/j.compscitech.2009.05.005

24. Schilling, M.; Bouchard, M.; Khanjian, H.; Learner, T.; Phenix, A. \& Rivenc, R. - Acc. Chem. Res., 43, p.888 (2010). PMid:20455567. http://dx.doi.org/10.1021/ar1000132

25. Chatterjee, P. K. \& Conrad, C. M. - J. Polym. Sci.: Part A-1, 6, p.3217 (1968).

26. Videki, B.; Klebert, S. \& Pokanzsky, B. - J. Polym. Sci.: Part B, 45, p.873 (2007). http://dx.doi.org/10.1002/polb.21121

27. Sommer, W. - "Plastic additives Handbook", cap.5, 2nd ed., Hanser Publishers, Munich (1987)

28. Cassu, S. N. \& Felisberti, M. I. - Quim. Nova, 28, p.255 (2005). http:// dx.doi.org/10.1590/S0100-40422005000200017

29. Alexander, S.; Entin-Wolhman, O. \& Orbach, R. - Phys. Rev. Part: B, 34, p.2726 (1986). http://dx.doi.org/10.1103/PhysRevB.34.2726

30. Dashora, P. \& Gupta, G. - Polymer., 37, p.231 (1996). http://dx.doi. org/10.1016/0032-3861(96)81092-5

31. Mahonty, A. K.; Wibowo, A.; Misra, M. \& Drzal L. T. - Polym. Sci. \& Eng., 43, p.1151 (2003).

32. Le Baillif, M. \& Oskman, K. - J. Therm. Comp. Mater., 22, p.115 (2009). http://dx.doi.org/10.1177/0892705708091608

Enviado: 29/06/11 Aceito: $12 / 09 / 11$ 